\title{
Using Drug Prescribing Patterns to Identify Stewards of Cost-Conscious Care
}

\author{
Nicole Michele Gastala, MD, Peter Wingrove, BS, Winston Liaw, MD, MPH, \\ Stephen Petterson, PhD, and Andrew Bazemore, MD, MPH
}

Purpose: To characterize family physicians (FPs) who are stewards of care by consistently prescribing omeprazole over esomeprazole.

Methods: Cross-sectional analysis of physicians prescribing omeprazole or esomeprazole under Medicare Part D in 2014.

Results: There was a regional trend with $49 \%$ of Western FPs but only $6 \%$ of Southern FPs rarely prescribing esomeprazole. Physicians had increased odds of being a steward if they worked with a care coordinator $(P<.001)$, at a patient-centered medical home $(P<.001)$, or in a large practice $(P<.001)$.

Conclusions: If these findings are replicated across multiple drugs, future outreach could be conducted based on provider prescribing patterns. (J Am Board Fam Med 2017;30:824-827.)

Keywords: Medicare, Prescription Drugs, Stewards

Responsible stewardship of health care expenditure is an important movement in an era of increasing costs. The Centers for Medicare and Medicaid Services publicly released comprehensive cost data for all medications in 2013 and 2014 under Medicare Part D. ${ }^{1,2}$ Brand-only esomeprazole was listed in the top 10 by cost despite the availability of generic therapeutic equivalent omeprazole that is well supported by medical literature. ${ }^{1-5}$ Although esomeprazole contains only the active S-enantiomer whereas omeprazole contains an equal mix of the S-Enantiomer and R-enantiomer, many studies show no significant difference in efficacy. ${ }^{3-5}$ Preferred and nonpreferred brand medications carry a higher total cost and copayment cost compared with generic medications under Medicare Part D prescription drug plans. ${ }^{6}$ Preferential prescribing of esomeprazole over omeprazole can result in a significant financial burden to Medicare Part D

This article was externally peer reviewed.

Submitted 5 February 2017; revised 12 May 2017; accepted 15 May 2017.

From Primary Health Care, Inc., Marshalltown, IA (NMG); Robert Graham Center for Policy Studies, Washington, DC (PW, WL, SP, AB).

Funding: none.

Conflict of interest: none declared.

Corresponding author: Nicole Michele Gastala, MD, Primary Health Care, Inc., 412 East Church Street, Marshalltown, IA 50158 (E-mail: reizinee@gmail.com). patients who paid an average of 10.5 times more for the branded medication. ${ }^{6}$ This data release presents an opportunity to evaluate prescribing habits of family physicians (FPs) to help target future evidence-based responsible stewardship interventions. The objective of this analysis was to identify and characterize stewards of cost-conscious prescribing.

\section{Methods}

We used Medicare Part D data from 2014 to examine prescribing patterns of physicians. This data lists which drugs each physician prescribed, how many days were prescribed, and the cost. We restricted this analysis to prescriptions of esomeprazole (introduced 2001) and generic prescriptions of omeprazole (introduced 1989). We defined stewards as physicians who prescribed esomeprazole less than $2 \%$ of the time rather than $0 \%$ as this accommodates rare events difficult to control by providers who may in normal circumstances never choose to prescribe esomeprazole. To exclude physicians who did not prescribe either drug frequently, we further restricted the analysis to physicians who prescribed 3650 days or more combined.

We used data from the American Board of Family Medicine to take a closer look at a sample of these physicians, board-certified FPs who recertified in 2014 or 2015. Information on practice size, working 
at a patient centered medical home (PCMH), and working alongside a care coordinator was self-reported as part of the recertification process. We excluded physicians initially certifying because we lacked data on their practice setting.

We obtained information about each physician's sex, country of training, year of graduation from medical school, and type of degree obtained from the American Medical Association (AMA) Physician Masterfile. Roughly $94 \%$ of the physicians in our sample were successfully matched with AMA records. Each physician's address was geocoded to determine region of the country and whether they practiced in a nonmetropolitan county as defined by a Rural-Urban Continuum Codes $\geq 4$.

We calculated the percent of physicians identified as stewards across a number of physician demographics and practice characteristics. $\chi^{2}$ tests were used to identify statistically significant differences with a threshold of $P<.05$ used to determine significance. Multivariate logit regression was used to assess the relationship between the likelihood each physician was a steward and our identified physician and practice characteristics.

\section{Results}

There were 15,688 FPs who both appeared in Part D Data for 2014 and recertified in 2014 or 2015. There were 8,792 FPs who met our threshold for significant prescribing; this subset accounted for $94.2 \%$ of all omeprazole and esomeprazole recorded days supplied in the Part D data.

Overall, $15.0 \%$ of the prescriptions were for esomeprazole. The median physician prescribed esomeprazole $13.0 \%$ of the time. As defined as prescribing only esomeprazole less than $2 \%$ of the time, 2001 (22.8\%) physicians met our criterion for being a steward (Figure 1). Approximately 27\% of women were stewards, but only $20 \%$ of men were. $(P<.001)$.

There were significant differences based on practice characteristics. Nearly half of physicians practicing in the West were stewards, but the same was true of only 1 in sixteen physicians from the South. $(P<.001)$ Urban FPs were more likely to not prescribe esomeprazole than their rural counterparts. $(P<.001)$ Physicians who practiced alongside 20 or more providers were 3 times as likely as solo practitioners. $(P<.001)$ Physicians who worked in PCMHs were approximately 50\% more likely to be stewards as those who did not
Figure 1. Distribution of physician prescribing behavior for choosing esomeprazole over omeprazole.

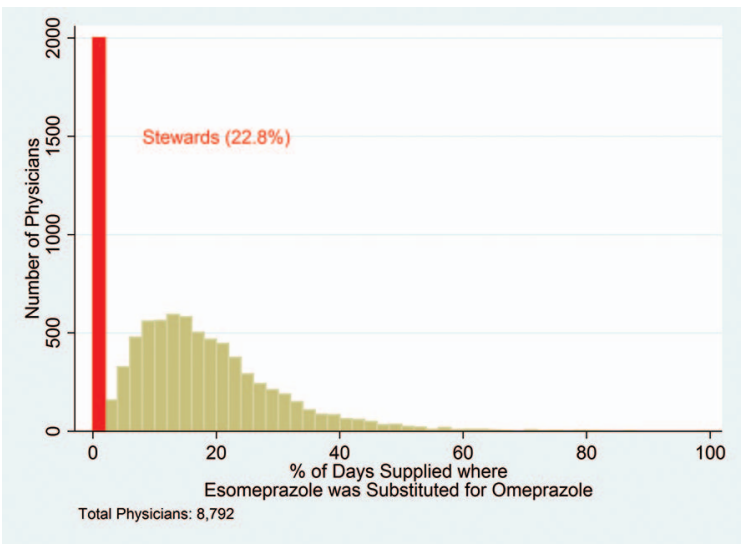

$(P<.001)$. The same was true for those working with care coordinators $(P<.001)$.

Multivariate logit regression revealed that many of these differences remain significant when holding other factors constant (Figure 2). Region alone explains much of the variation. Compared with physicians in the Midwest, Southern physicians were one fifth as likely to be stewards $(P<.001)$, and physicians in the Northeast were a little more than half as likely. In contrast, physicians practicing in the West were roughly 2.5 times as likely to be $(P<.001)$. Practice size was also a key determinant. Physicians at medium and large practices were $63 \%(P<.001)$ and $145 \%$ $(P<.001)$ more likely respectively to be stewards than solo practitioners. Independent of practice size, working with a care coordinator $(P<.001)$ or in a PCMH $(P<.001)$ each increased the likelihood of being a steward by approximately $50 \%$. The effects of demographics were more modest with women being a third more likely than men to be stewards. Physicians who graduated medical school before 1980 were less likely to be stewards than those graduating since 2000.

\section{Discussion}

In this analysis of Medicare Part D data, we found that $23 \%$ of our sample are prescribing stewards. Women, living in the West, and working in large practices that are PCMHs are associated with stewardship. Efforts to help physicians and patients engage in evidence-based conversations aimed at wise management and just distribution of finite resources were created with programs such as the Choosing Wisely campaign by the American Board 
Figure 2. Practice characteristics and other factors that increase likelihood of being a steward of health care expenditure as defined as prescribing generic omeprazole over esomeprazole (Nexium).

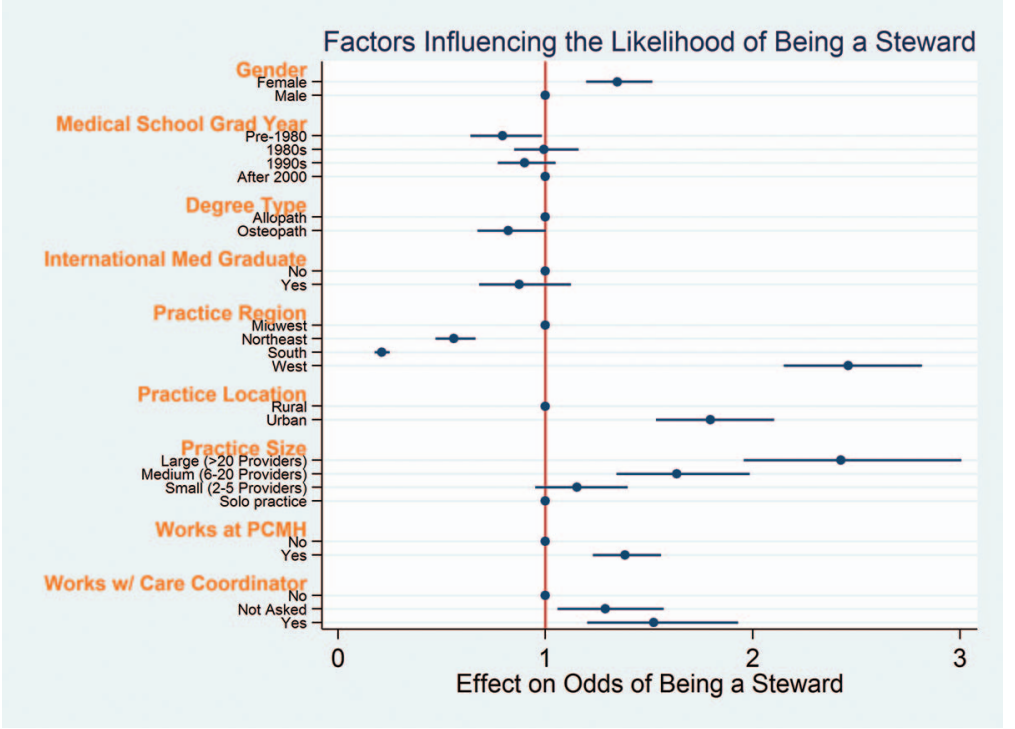

of Internal Medicine Foundation. ${ }^{7,8}$ The campaign consists of recommendations based on topics of care but has not targeted specific geographic areas or populations based on physician or practice characteristics related to increased expenditure.

Although more research needs to be conducted, future outreach programs could be directed based on practice or provider specific data to improve prescribing habits. Academic detailing through evidence-based information by specially trained noncommercial outreach educators to physician offices has been shown to be effective in more than 60 randomized controlled trials and could be targeted to specific locations or practice characteristics from future studies such as this. ${ }^{9}$ Furthermore, with the emergence of health care systems, there are opportunities for information feedback where physicians could be rewarded for prescribing drugs optimally. ${ }^{9}$

To see this article online, please go to: http://jabfm.org/content/ 30/6/824.full.

\section{References}

1. Centers for Medicare \& Medicaid Services (CMS). CMS releases prescriber-level Medicare data for first time. Baltimore, MD, Apr 30, 2015. Available from: https://www.cms.gov/Newsroom/MediaRelease Database/Fact-sheets/2015-Fact-sheets-items/201504-30.html. Accessed Mar 17, 2016.

2. Centers for Medicare \& Medicaid Services (CMS). Part D Prescriber Data CY 2014. Baltimore, MD,
Aug 18, 2016. Available from: https://www.cms.gov/ Research-Statistics-Data-and-Systems/StatisticsTrends-and-Reports/Medicare-Provider-ChargeData/PartD2014.html. Accessed Oct 17, 2016.

3. Congressional Budget Office. Effects of using generic drugs on medicare's prescription drug spending. Washington, DC, Sep 15, 2010. Available from: https://www.cbo.gov/publication/21800. Accessed Dec 16, 2016.

4. Teng M, Khoo AL, Zhao YJ, et al. Meta-analysis of the effectiveness of esomeprazole in gastroesophageal reflux disease and Helicobacter pylori infection. J Clin Pharm Ther 2015;40:368-375.

5. McDonagh MS, Carson S, Thakurta S. Drug class review: Proton pump inhibitors: Final report update 5. Portland, OR: Oregon Health \& Science University; May 2009. Available from: https://www.ncbi. nlm.nih.gov/books/NBK47260/.

6. Gastala NM, Wingrove P, Gaglioti A, Petterson S, Bazemore A. Medicare Part D: Patients bear the cost of 'me too' brand-name drugs. Health Affairs 2016; 35:1237-1240.

7. Good Stewardship Working Group. The "top 5" lists in primary care: Meeting the responsibility of professionalism. Arch Intern Med 2011;171:13851390.

8. Choosing Wisely: An initiative of the ABIM foundation. Philadelphia, PA. Available from: http:// www.choosingwisely.org. Dec 16, 2016.

9. Kesselheim AS, Avorn J, Sarpatwari A. The high cost of prescription drugs in the Unites States origins and prospects for reform. JAMA 2016;316:858-871. 


\begin{tabular}{|c|c|c|}
\hline \multicolumn{3}{|c|}{ Bivariate Table: Demographics } \\
\hline & Column (\%) & Steward (\%) \\
\hline \multicolumn{3}{|l|}{ Sex ${ }^{*}$} \\
\hline Female & 32.9 & 27.3 \\
\hline Male & 67.1 & 20.2 \\
\hline \multicolumn{3}{|l|}{ Race* $^{*}$} \\
\hline Asian & 11.6 & 28.9 \\
\hline Black or African American & 5.2 & 18.2 \\
\hline Other & 1.4 & 36.5 \\
\hline White & 81.7 & 21.7 \\
\hline \multicolumn{3}{|l|}{ Graduation Year* } \\
\hline Pre-1980 & 12.3 & 17 \\
\hline $1980 s$ & 33.8 & 22.8 \\
\hline $1990 \mathrm{~s}$ & 33.7 & 22.5 \\
\hline After 2000 & 20.2 & 25.7 \\
\hline \multicolumn{3}{|l|}{ Degree Type } \\
\hline Allopath & 91 & 22.8 \\
\hline Osteopath & 9 & 20.2 \\
\hline \multicolumn{3}{|c|}{ International Medical Graduate (Non-US or Canada) } \\
\hline No & 94.8 & 22.5 \\
\hline Yes & 5.2 & 23 \\
\hline \multicolumn{2}{|c|}{ Bivariate Table: Practice Characteristics } & Steward (\%) \\
\hline \multicolumn{3}{|l|}{ Practice Region* } \\
\hline Midwest & 28.8 & 25.8 \\
\hline Northeast & 15.3 & 17.2 \\
\hline South & 35.2 & 6.6 \\
\hline West & 20.7 & 49.1 \\
\hline \multicolumn{3}{|l|}{ Practice Location* } \\
\hline Rural & 19.7 & 13.9 \\
\hline Urban & 80.3 & 24.7 \\
\hline \multicolumn{3}{|l|}{ Practice Size* } \\
\hline Large ( $>20$ providers) & 13.8 & 41.2 \\
\hline Medium (6 to 20 providers) & 30.2 & 27.3 \\
\hline Small ( 2 to 5 providers) & 39.9 & 16.3 \\
\hline Solo practice & 16 & 13.2 \\
\hline \multicolumn{3}{|l|}{$\mathrm{PCMH}^{*}$} \\
\hline Autofill for skipped question & 0.1 & 15.4 \\
\hline No & 64.5 & 19 \\
\hline Yes & 35.3 & 29.1 \\
\hline \multicolumn{3}{|l|}{ Works w/ Care Coordinator* } \\
\hline No & 11.7 & 15.4 \\
\hline \multirow[t]{2}{*}{ Not asked } & 75.1 & 22.5 \\
\hline & 13.2 & 29.4 \\
\hline
\end{tabular}

Asterisk signifies chi-2 * $(P<.001)$.

$\mathrm{PCMH}$, patient centered medical home. 